\title{
Modifications de la liste des analyses au 1er janvier 2015
}

Susanne Christen

Dr, FMH, division Tarifs et conventions pour la médecine ambulatoire en Suisse
Correspondance:

FMH / division Tarifs et

conventions pour la médecine ambulatoire en Suisse

Frohburgstrasse 15

CH-4600 Olten

Tél. 0313591230

Fax 0313591238

tarife.ambulant[at]fmh.ch
Le Département fédéral de l'intérieur (DFI) a décidé de modifier la liste des analyses concernant le laboratoire au cabinet médical au $1^{\text {er }}$ janvier 2015. Vous avez déjà pu lire des informations à ce sujet dans l'éditorial du Bulletin des médecins suisses du 12 novembre 2014 (numéro 46) du Dr Ernst Gähler, vice-président de la $\mathrm{FMH}$, responsable du département Tarifs et conventions pour la médecine ambulatoire en Suisse.

Ces modifications concernent uniquement le laboratoire au cabinet. D'une part, certaines analyses du diagnostic en présence du patient au laboratoire du cabinet (désormais intitulées «analyses rapides») font l'objet d'une nouvelle tarification, et d'autre part, certaines positions existantes en lien avec la taxe de présence et les suppléments connaissent également quelques modifications.

\section{Positions générales}

La liste des analyses est divisée en plusieurs chapitres. Le chapitre 4 «Positions générales» comprend les positions qui ne doivent être utilisées que pour les traitements ambulatoires. Dans ce chapitre, les modifications sont les suivantes:

La taxe de présence 4707.00 a été complètement supprimée pour le laboratoire au cabinet médical.

Le supplément pour chaque analyse présentant le suffixe $C, 4707.10$, n'est désormais applicable plus que pour:

- les analyses du chiffre 5.1.2.2.2 «Analyses complémentaires»,

- les analyses du chiffre 5.1.3 «Analyses pour les médecins avec certains titres postgrades» et

- la position 1735.00 troponine, qui fait partie des analyses sous chiffre 5.1.4 «Analyses effectuées par les médecins dans le cadre d'une consultation à domicile».

\begin{tabular}{|c|c|c|c|c|}
\hline \multirow[t]{2}{*}{ Analyses } & \multicolumn{3}{|c|}{ Chiffre 5.1.2.2.1 LA Chiffre 5.1.2.2.2 LAChiffre 5.1.4 LA } & \multirow[b]{2}{*}{$\begin{array}{l}\text { Chiffre } 5.1 .3 \text { LA } \\
\text { Liste élargie pour } \\
\text { les médecins avec } \\
\text { certains titres } \\
\text { postgrades }\end{array}$} \\
\hline & Analyses rapides & $\begin{array}{l}\text { Analyses } \\
\text { complémentaires }\end{array}$ & $\begin{array}{l}\text { Analyses dans le cadre } \\
\text { d'une consultation à } \\
\text { domicile }\end{array}$ & \\
\hline Tarif & $\begin{array}{l}\text { nouveau tarif } \\
\text { (Analyses rapides) }\end{array}$ & $\begin{array}{l}\text { tarif actuel (aucun } \\
\text { changement) }\end{array}$ & $\begin{array}{l}\text { en partie nouveau tarif } \\
\text { (Analyses rapides) }\end{array}$ & $\begin{array}{l}\text { tarif actuel (aucun } \\
\text { changement) }\end{array}$ \\
\hline Diagnostic en présence & exigé & exigé & exigé & exigé \\
\hline 4707.00 Taxe de présence & supprimé & supprimé & supprimé & supprimé \\
\hline 4707.10 Supplément suffixe (avec C) & supprimé & applicable & seul pour $n^{\circ}$ LA 1735.00 & applicable \\
\hline 4707.20 Supplément suffixe (sans C) & supprimé & applicable & supprimé & applicable \\
\hline 4708.00 Supplément de transition & supprimé & supprimé & supprimé & supprimé \\
\hline
\end{tabular}

Le supplément pour chaque analyse ne présentant pas le suffixe $C, 4707.20$, n'est désormais cumulable plus qu'avec les analyses du chiffre 5.1.2.2.2 «Analyses complémentaires» et celles du chiffre 5.1.3 «Analyses pour les médecins avec certains titres postgrades».

Le supplément de transition 4708.00 est supprimé sans contrepartie au $1^{\text {er }}$ janvier 2015.

\section{Annexes à la liste des analyses}

Le chapitre 5 de la liste des analyses «Annexes à la liste des analyses», chiffre 5.1 annexe A, énumère les analyses fournies dans le cadre des soins de base.

Les listes partielles 1 (chiffre 5.1.2.2) et 2 (chiffre 5.1.2.3) de la liste des analyses 2014 sont remplacées par les listes suivantes:

- 5.1.2.2 Analyses pour le laboratoire de cabinet médical

\section{- 5.1.2.2.1 Liste des analyses rapides}

\section{- 5.1.2.2.2 Liste des analyses complémentaires}

- 5.1.2.3 Analyses pour les laboratoires d'hôpitaux au sens de l'art. 54 al. 1 let. b OAMal et pour les laboratoires d'hôpitaux au sens de l'art. 54 al. 1 let. c OAMal en liaison avec l'art. 54 al. 2 OAMal

- 5.1.2.4 Analyses pour les officines de pharmaciens (art. 54 al. 1 let. c OAMal)

Les listes suivantes sont maintenues:

- 5.1.3 Liste élargie des analyses pour les médecins avec certains titres postgrades

- 5.1.4 Analyses effectuées par les médecins dans le cadre d'une consultation à domicile. Cette liste n'a pas été remplacée mais adaptée conformément aux «analyses rapides». 


\section{Diagnostic en présence du patient}

Les conditions d'admission suivantes s'appliquent aux laboratoires de cabinets médicaux conformément à l'art. 54 al. 1 let. a OAMal (inchangées): Les analyses dans le cadre des soins de base sont effectuées pour les besoins du médecin, c'est-à-dire pour ses propres patients. Le résultat des analyses est en principe disponible au cours de la consultation, c'est-à-dire en présence du patient (diagnostic en présence du patient). Cela signifie que le diagnostic en présence du patient est

\section{Quelques liens utiles}

L'éditorial du Dr Ernst Gähler, vice-président de la FMH, responsable du département Tarifs et conventions pour la médecine ambulatoire en Suisse, «Laboratoire au cabinet médical: bémol dans la revalorisation tant attendue de la médecine de famille», BMS $\mathrm{n}^{\circ}$ 46: www.fmh.ch $\rightarrow$ Tarifs ambulatoires $\rightarrow$ Publications $\rightarrow$ Bulletin des médecins suisses - Autres publications: www.fmh.ch/files/pdf15/BMS-46.pdf

Les questions fréquemment posées (FAQs) sur le laboratoire au cabinet: www.fmh.ch $\rightarrow$ Tarifs ambulatoires $\rightarrow$ Publications $\rightarrow$ Recommandations pour la facturation / FAQ: www.fmh.ch/files/pdf15/20141007-FAQ-Labor-FR.pdf

La liste des analyses, les modifications au 1er janvier 2015 et la Helpdesk de l'OFSP: www.bag.admin.ch $\rightarrow$ Thèmes $\rightarrow$ Assurance-maladie $\rightarrow$ Tarifs et prix $\rightarrow$ Liste des analyses: www.bag.admin.ch/themen/krankenversicherung/00263/00264/04185/ index.html?lang=fr exigé aussi bien pour les analyses rapides que pour toutes les autres analyses.

\section{Tarification}

Dans la liste des analyses 2014, toutes les positions sont tarifées de manière identique. Cependant, la rémunération des analyses diffère selon l'endroit où elles sont fournies (laboratoire mandaté, laboratoire d'hôpital, laboratoire du cabinet ou pharmacie) en raison des différences d'application des 9 positions générales complémentaires.

A partir du $1^{\text {er }}$ janvier 2015, les 33 analyses de la liste des «analyses rapides» seront tarifées différemment. Pour savoir s'il s'agit ou non d'une position correspondant à une «analyse rapide», il convient de consulter le sixième chiffre du numéro de la position. Exemple: l'analyse Potassium porte le numéro 1479.01 dans la liste des «analyses rapides», et le numéro 1479.00 dans les autres listes. Suite à la nouvelle tarification, toutes les positions générales complémentaires pour les «analyses rapides» ont été supprimées.

Le tableau de la page précédente donne un aperçu des modifications de la liste des analyses relatives au laboratoire du cabinet médical à partir du $1^{\text {er }}$ janvier 2015 .

\title{
L'afffliation à la FMH n'a que
}

\section{des avantages.}

\author{
Nous vous soutenons grâce à une politique \\ engagée et des services attrayants: ligne \\ d'assistance tarifaire, renseignements juri- \\ diques, contrats-type.
}

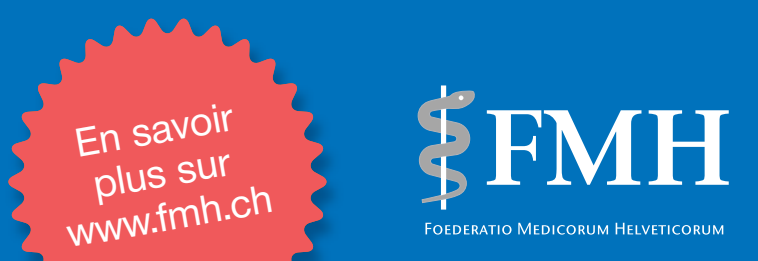

